\title{
Hierarchical Market Competition in a Duopoly Super Wi-Fi Spectrum Market
}

\author{
Hyoil Kim, Member, IEEE, Jaehyuk Choi, Member, IEEE, and Kang G. Shin, Fellow, IEEE
}

\begin{abstract}
Super Wi-Fi refers to Wi-Fi-like Internet access via spectrum white spaces (WS), which is expected to enhance today's Wi-Fi thanks to the superior propagation characteristics of the WS compared to ISM/UNII bands. A Super Wi-Fi wireless service provider (WSP) dynamically leases and opportunistically utilizes a licensed band while it is temporarily unoccupied by its (licensed) primary users (PUs). The PUs' spectrum usage pattern presents time-varying spectrum availability, thus necessitating eviction of in-service customers upon return of PUs where the evicted customers are compensated with partial reimbursement of their service charge. This paper investigates the dynamics of a duopoly Super Wi-Fi market where two co-located WSPs compete for leasing better quality channels and for setting competitive service price to entice more customers. The channel quality is measured by the PUs' utilization factor (smaller the better). Since higher quality channels possess more WS incurring larger channel leasing cost, a WSP should strike a balance between channel quality and service tariff in maximizing its profit. The market competition is modeled as hierarchical noncooperative price- and quality-games, and their Nash Equilibria (NE) are derived. In addition, we investigate the impact of differentiated reimbursement rates and limited channel availability. Finally, we demonstrate the tradeoffs among leasing cost, customer arrival rate, and channel characteristics via numerical analyses.
\end{abstract}

Index Terms-Super Wi-Fi, dynamic spectrum access, duopoly spectrum market, time-varying spectrum availability

\section{INTRODUCTION}

$\mathbf{T}$ HE ADVENT of dynamic spectrum access (DSA) has paved the way to improve the utilization of scarce spectrum resources. DSA enables unlicensed users, called secondary users (SUs), to opportunistically access spectrum white spaces (WS) of the under-utilized legacy spectrum in the absence of licensed users, called primary users (PUs); DSA has become realizable with the cognitive radio (CR) technology. This concept of reusing the legacy spectrum is expected to stimulate new applications in commercial, public, and military networks.

The commercial use of DSA has been encouraged by the FCC's final ruling released in 2010 [2] that allows unlicensed

Manuscript received: November 18, 2012; revised date: April 4, 2013. A preliminary version of this work was presented at the IEEE INFOCOM 2011 [1]. The work described in this paper was supported in part by the year of 2011 Research Fund of the UNIST, and the Basic Science Research Program through the NRF of Korea funded by the Ministry of Education, Science and Technology (2012R1A1A1014648 and 2012R1A1A1014755).

H. Kim is with the School of Electrical and Computer Engineering, the Ulsan National Institute of Science and Technology (UNIST), Ulsan 689798, Korea (e-mail: hkim@unist.ac.kr).

J. Choi is with the Department of Software Design and Management, Gachon University, Seongnam 461-701, Korea (e-mail: jchoi@gachon.ac.kr).

K. G. Shin is with Real-Time Computing Laboratory, The University of Michigan, Ann Arbor, MI 48109, USA (e-mail: kgshin@eecs.umich.edu).

Digital Object Identifier 10.1109/JSAC.2013.131135. radio operation in the DTV bands by fixed and portable devices. The fixed devices represent high-power stationary transceivers in rural areas for the last-mile services (e.g., IEEE 802.22 [3]), and the portable devices represent short-range transceivers in urban areas for WiFi-like Internet access which is often referred to as Super Wi-Fi [4]-[6]. Super Wi-Fi is considered a promising application of DSA to enhance the network coverage and mitigate in-network collision compared to today's Wi-Fi, thanks to the superior propagation characteristics of the legacy licensed bands such as the wall-penetrating ability of the UHF/VHF bands [7]. ${ }^{1}$

A Super Wi-Fi network is operated by a wireless service provider (WSP) that temporarily leases a licensed spectrum band (or channel) via the multi-winner periodic spectrum auction managed by the spectrum broker (SB) and provides the CR customers Internet access by utilizing the leased spectrum. Once the leasing term ends, all channels are returned to the licensees and the WSPs must participate in the auction again. The concept of Super Wi-Fi is illustrated in Fig. 1.

Following the model of preemptive spectrum lease in [8], it is assumed that a leased channel can be preempted by its PUs during the leasing term. Specifically, a channel is modeled as an ON/OFF source [9], [10] as shown in Fig. 2, where ON periods imply PUs' activities and OFF periods represent PUs' absence or existence of spectrum WS. Then, the lessee (i.e., WSP) of the channel is allowed to access the OFF periods only and the licensee can collect the channel leasing fee only for those OFF periods. This model is a realization of the private commons [11] which is believed as a viable option to benefit both PUs and SUs because PUs can still access their channels during the leasing period while making extra profit.

In this paper, we investigate price and quality competitions in a duopoly Super Wi-Fi market ${ }^{2}$ between two co-located WSPs. Each WSP leases a licensed channel with time-varying availability due to the PUs' ON/OFF channel-usage patterns, and hence, upon (re)appearance of PUs, the WSP should evict all in-service SUs from its network (called channel vacation) to protect the PUs. ${ }^{3}$ It is assumed that the WSP compensates the evicted customers by reimbursing (part of) their service charges for the sake of customer satisfaction. Therefore, a WSP should lease a channel with proper quality in terms

\footnotetext{
${ }^{1}$ Note that Super Wi-Fi is different from the CR network in the ISM bands because the former has to protect incumbent users with high priority while the latter shares the spectrum with other types of unlicensed users.

${ }^{2}$ Here we consider duopoly for the ease of analysis, but the duopoly scenario provides enough insight into the network dynamics of Super WiFi.

${ }^{3}$ Although the WSP can also keep customers in the system while suspending its service during ON periods, it cannot achieve seamless service provisioning.
} 


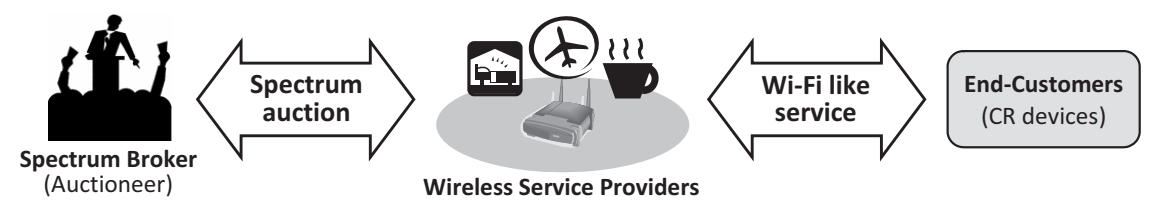

Fig. 1. The three-tier Super Wi-Fi market

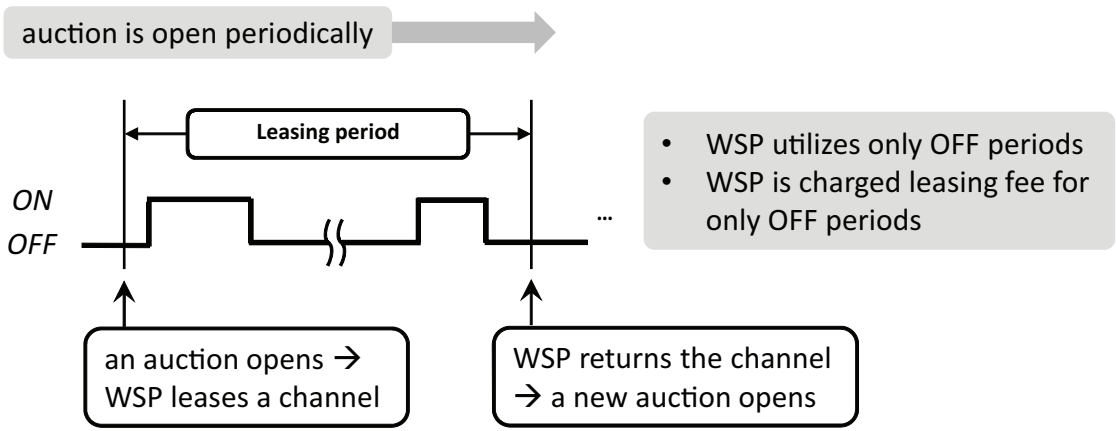

Fig. 2. The preemptive lease model with ON-OFF channels

of PUs' channel utilization, that incurs less eviction and a smaller leasing cost. Each WSP should also determine the optimal pricing strategy in terms of the service tariff, because a higher price than its competitor will attract less customers and generate less profit.

The contribution of this paper is three-fold. First, we model the interaction between WSPs as a hierarchical noncooperative game with price and quality competitions while accounting for time-varying spectrum availability. The existing gametheoretic approaches to the dynamic spectrum market [12][16] have been limited to consistently-available channels, and to the best of our knowledge, this is the first attempt to incorporate the effect of time-varying spectrum availability in the game-theoretic framework. Second, we analyze the market dynamics using a Markov chain and derive the Nash Equilibria (NE) of the price and quality competitions. In addition, we investigate the impact of service price and reimbursement rates on customer service preference for price competition. As to quality competition, we present the existence of market entry barrier and discuss the case of a limited number of channels in the auction. Finally, we perform extensive numerical analyses to provide important insights into the Super Wi-Fi market.

The rest of the paper is organized as follows. Section II reviews related work, and Section III introduces the system model and assumptions used in the paper. Section IV describes the hierarchical game framework and models the system as a Markov Chain from which the profit and cost functions are derived. Then, Sections V and VI formulate and analyze the price and quality competitions, respectively. Section VII also investigates the impact of heterogeneous reimbursement rates upon eviction of users. The market dynamics under various network conditions are studied via numerical analyses in Section VIII, and the paper concludes with Section IX.

\section{RELATED WORK}

Market competition between WSPs in DSA networks has been studied in the literature based on various game theoretic frameworks. Jia and Zhang [12] investigated price and capacity competition between WSPs where customers choose a WSP based on a pre-determined utility, not driven by price. Duan et al. [13] considered channel leasing and price competition and derived threshold-type pricing rules assuming a fixed spectrum leasing cost. Our work, however, is built upon spectrum auction literature [12], [17] by modeling leasing cost dependent on the total spectrum demand in auction. Zhu et al. [14] introduced a hierarchical spectrum market with spectrum brokers and secondary service providers, where dynamic spectrum leasing and service selection are investigated. Kasbekar et al. [18] considered a hierarchical game between two spectrum market layers, and analyzed the interaction between the regulator, service providers and mobile subscribers.

None of the above, however, captured the impact of timevarying spectrum availability according to PUs' spectrum usage while this paper does that. In a similar vein, Kasberkar et al. [15] analyzed the case where the availability of licensed channels is determined with some probability, but they assumed that no channel-state transition occurs during a lease term. Although Duan et al. [16] studied a leasingpricing problem of a cognitive network operator where PUs' spectrum usage varies with time slots, spectrum availability is still assumed static within each slot.

\section{System Model And Assumptions}

In this section, we describe the system model and assumptions used throughput the paper.

\section{A. Channel Model}

A channel represents one of the legacy spectrum bands (with WS) being utilized by PUs. Although the FCC currently considers WS in TV bands in the beginning of DSA commercialization, our channel model is designed as general as possible to be capable of describing Super Wi-Fi based on various types of WS. 
We assume WSP $i(i=1,2)^{4}$ leases a channel with capacity $C_{i}$ from the auction, which is modeled as an ON/OFF source as shown in Fig. 2. This type of channel model has been used in many applications [9], [10], since it can describe PUs' signal activity patterns in a continuous time-domain. We also assume $\mathrm{ON}$ and $\mathrm{OFF}$ periods are exponentially distributed with rate $\Lambda_{i}^{O N}$ and $\Lambda_{i}^{O F F}$, respectively, and then channel utilization by PUs (denoted by $u_{i}$ ) is given as

$$
u_{i}=\frac{1 / \Lambda_{i}^{O N}}{\left(1 / \Lambda_{i}^{O N}+1 / \Lambda_{i}^{O F F}\right)}=\frac{\Lambda_{i}^{O F F}}{\left(\Lambda_{i}^{O N}+\Lambda_{i}^{O F F}\right)} .
$$

Estimation of the channel parameters $\left(\Lambda_{i}^{O N}, \Lambda_{i}^{O F F}, u_{i}\right)$ and detection of ON/OFF periods can be achieved via spectrum sensing [9], which is outside the scope of this paper. In addition, we assume homogeneous channel capacities such that $C_{i}=C, \forall i$, which holds when the same type of licensed bands (e.g., DTV channels) are considered.

\section{B. Auction Model}

We consider a multi-winner periodic spectrum auction [19], [20] as shown in Fig. 2, where the leasing period is assumed to be long relative to the sojourn times of $\mathrm{ON}$ and OFF periods to enable the steady-state analysis of the behavior of WSPs according to ON-OFF transitions. For example, for TV WS, a leasing period could be as long as a few days or weeks since TV broadcasting usually lasts for several hours.

In our auction model, WSP $i$ leases a channel with the utilization of $u_{i}$ and pays the leasing fee of $L_{i}$ per unittime. To describe the form of $L_{i}$, we introduce the concept of effective channel capacity, denoted by $C_{i}^{e f f}$ and given as

$$
C_{i}^{e f f}=\left(1-u_{i}\right) C
$$

which implies the total effective amount of the leased spectrum available for WSP $i$. Then, the DSA auction model in [12], [17] has shown that the unit price function $l$, i.e., the leasing price per unit-bandwidth, is given as ${ }^{5}$

$$
l=\gamma_{1}\left(C_{i}^{e f f}+C_{-i}^{e f f}\right)^{\gamma_{2}}, \gamma_{1}>0, \gamma_{2} \geq 1,
$$

which is a positive, non-decreasing and convex function of $\left(C_{i}^{e f f}+C_{-i}^{e f f}\right)$. That is, the leasing cost depends on the total spectrum demand in the auction market, where $\gamma_{1}$ is the baseline cost when the total demand is unity. In addition, the leasing cost increases faster than proportionally to the total demand (i.e., $C_{i}^{e f f}+C_{-i}^{e f f}$ ) due to the competition between WSPs for the limited spectrum resources auctioned off, where the degree of competition is described by $\gamma_{2}$. Finally, the leasing cost function $L_{i}$ of WSP $i$ is given as

$$
L_{i}=C_{i}^{e f f} \cdot l=\bar{\gamma}_{1}\left(1-u_{i}\right)\left(2-u_{i}-u_{-i}\right)^{\gamma_{2}},
$$

where $\bar{\gamma}_{1}=\gamma_{1} C^{1+\gamma_{2}}>0$ is the normalized $\gamma_{1}$.

\footnotetext{
${ }^{4}$ We use $i$ as a WSP index and use $-i$ to denote WSP $i$ 's competitor.

${ }^{5}$ Note that in [12], [17] $l$ is derived for the total capacity of spectrum, while in our case $l$ is derived for effective capacity of spectrum. The reason is that spectrum in [12], [17] is assumed always available whereas our model considers opportunistic usage of spectrum according to $u_{i}$.
}

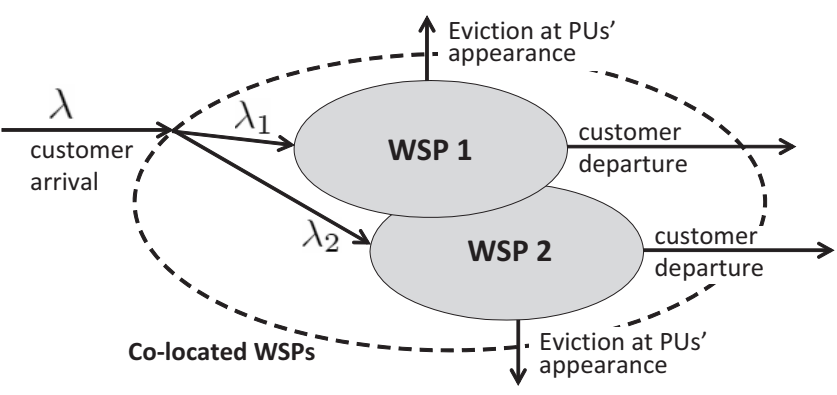

Fig. 3. A duopoly Super Wi-Fi network

\section{Service Model}

1) Customer arrivals and departures: We assume that customer arrivals follow a Poisson distribution with rate $\lambda$ and their service time is exponentially distributed with mean $1 / \mu$. A customer arrival represents a customer's connection request to a Super Wi-Fi WSP and the service time represents the customer's cell sojourn time, where both features are defined not at the packet level but at the session level. Connection-level arrivals are known to be modeled well as a Poisson process [21]. The cell sojourn time, however, has been modeled with various distributions, such as exponential [22], heavy-tailed Pareto [23], hyper-exponential [24], and Gamma distribution [25], where there exists a tradeoff between modeling accuracy and mathematical tractability.

In this paper, exponential service time is assumed because it not only approximates the reality but also makes it tractable to analyze a WSP's system in Section IV which enables our game-theoretic analysis in Sections $\mathrm{V}$ and VI to obtain a general insight of the market competition.

We define $\rho:=\lambda / \mu$ and assume $0<\rho<1$. In addition, the bulk customer arrivals are split into two flows such that WSP $i$ has arrival rate $\lambda_{i}$ and $\lambda=\lambda_{i}+\lambda_{-i}$ as shown in Fig. 3, for which we also define $\rho_{i}:=\lambda_{i} / \mu$.

We assume that each customer demands the bandwidth of $B(B \leq C)$ where $C$ is a multiple of $B .^{6}$ Then, by defining $\alpha:=C / B(\geq 1)$, which is a positive integer, we can have up to $\alpha$ concurrent in-service customers at a WSP.

2) Service price: An in-service customer at WSP $i$ is charged at the rate of $p_{i}$ per unit-time, where it is assumed $\underline{\Pi}_{i} \leq p_{i} \leq \bar{\Pi}$. $\bar{\Pi}$ is referred to as the monopoly price above which WSP $i$ would attract no customers because customers may not choose the 'best-effort' CR service if the 'guaranteed' legacy service offers more competitive price. Therefore, $\bar{\Pi}$ is determined by the tariff of the legacy services (e.g., 3G networks) and is assumed given a priori. On the other hand, $\underline{\Pi}_{i}$ is called the marginal price under which the WSP cannot make profit due to the channel leasing and user eviction costs. $\underline{\Pi}_{i}$ will be derived in Section V.

The monopoly price can also be modeled to be qualitysensitive, such that $\bar{\Pi}$ becomes smaller if customers experience more frequent evictions. In such a case, CR WSPs need to lease good-quality channels to maintain larger $\bar{\Pi}$, which is left as our future work.

\footnotetext{
${ }^{6}$ It is possible to consider multi-class customers with varying demands for user-level QoS at the expense of increased complexity in the Markov chain analysis, in Section IV.
} 


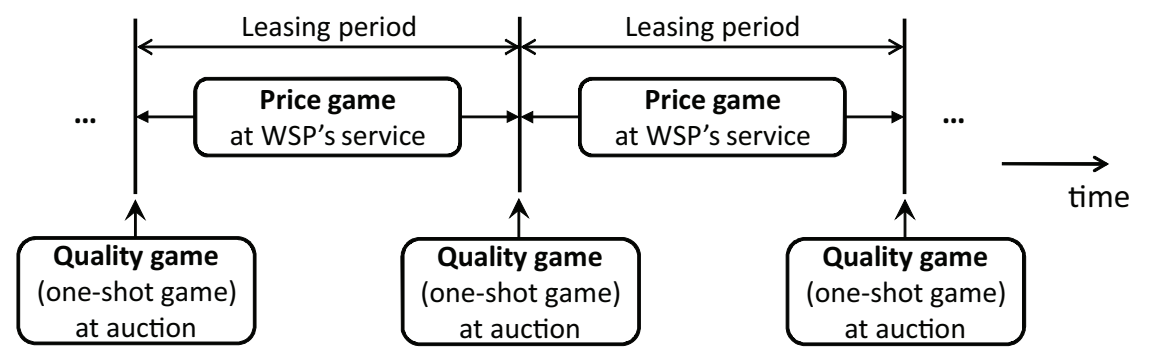

Fig. 4. Price Sub-Game and Quality Full-Game

3) Service discovery and preference: Each WSP is assumed to broadcast beacons via its leased channel while it is idle (i.e., OFF), to indicate that its network is available for service. Then, an arriving customer scans a predetermined range of channels (e.g., a list of DTV channels reserved for Super WiFi) to find in-service WSPs at its location, and selects the one with the lowest advertised service price. In case the WSP chosen by the customer is fully occupied by SUs, the customer leaves the Super Wi-Fi site. ${ }^{7}$

When a WSP's channel is occupied by PUs (i.e., ON), it cannot broadcast beacons and no customer visits its network. Therefore, even if $p_{i}>p_{-i}$, WSP $i$ can have customer arrivals while its channel is idle and WSP $-i$ 's channel is busy.

4) User eviction: Upon appearance of PUs, a WSP should evict all in-service customers from the network to protect the PUs. ${ }^{8}$ Each evicted user is compensated by the reimbursement of $I=\beta \cdot p_{i} / \mu, \beta>0$, i.e., $\beta$ times the average service charge without eviction. We also assume $\beta \leq 1$ to make the compensation upper-bounded by what customers pay on average. In addition, we assume $\Lambda_{i}^{O F F} / \mu=(1 / \mu) /\left(1 / \Lambda_{i}^{O F F}\right)<1$, because it is not beneficial to lease a channel that cannot serve even a single session in an OFF period. It is also possible to differentiate the reimbursement rate $\beta$ between WSPs (i.e., $\beta_{i}$ ) so that the service tariff is described by $\left(p_{i}, \beta_{i}\right)$. Section VII will discuss the details of such a scenario.

\section{Hierarchical Game and Markov Model of MARKET COMPETITION}

The market competition between WSPs is modeled as a two-stage noncooperative game, consisting of the price and quality games. The quality game is performed periodically at every auction, where each WSP competes for the desired quality of channel to lease described by $u_{i}$. The quality game is a one-shot game such that WSPs cannot return or change the leased channel before the current leasing term ends. During a leasing term, two WSPs perform the price game to determine the optimal price $p_{i}$ for their maximal profit. The quality game is also called a full-game in the sense that the optimal quality of spectrum is determined by assuming the NE prices of the

\footnotetext{
${ }^{7}$ This is a reasonable assumption since the WSP-customer relationship is volatile due to the flexible design of $\mathrm{CR}$ devices. That is, CR customers may choose different services (e.g., Wi-Fi, 3G) by reconfiguring themselves if the desired WSP's service is not instantly available.

${ }^{8} \mathrm{We}$ assume the evicted customers will use alternative services such as Wi-Fi or $3 \mathrm{G}$ networks, due to the flexibility of the CR devices.
}

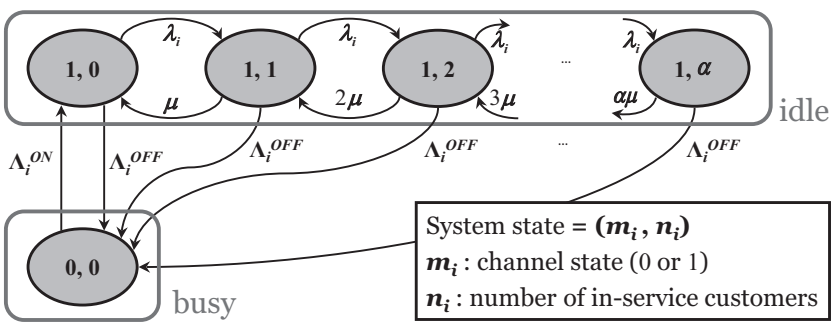

Fig. 5. State transition of WSP $i$ 's system

two WSPs achieved at a price sub-game. The hierarchy of price and quality games is illustrated in Fig. 4.

As the main objective of a WSP is to maximize its profit, the profit function must be analytically derived before investigating the price and quality competitions. To derive the profit, we define the system state of WSP $i$ as $s_{i}=\left(m_{i}, n_{i}\right)$ where $m_{i}=0$ if channel is busy and $m_{i}=1$ otherwise, and $n_{i}$ is the number of in-service customers with $n_{i} \in\left[0, m_{i} \alpha\right]$. Then, the system can be modeled as a Markov Chain under the assumption of Poisson arrivals, exponential service times, and exponential $\mathrm{ON}$ and OFF periods.

Fig. 5 illustrates the state-transition diagram of the Markov Chain. The horizontal transitions represent the state transitions by the customer arrivals and departures, and the vertical transitions represent the state transitions due to ON/OFF channel state changes. A customer arrival is accepted by the system if $n_{i}<m_{i} \alpha$. When an idle channel becomes busy, all $n_{i}$ customers are evicted from the system.

Although a numerical analysis can be used to find the stationary probability of the system, a closed-form is preferred for the analysis of the price and quality games to gain an insight in the form of the price and quality NEs. Therefore, we approximate the original Markov Chain by applying a state decomposition technique introduced in [26].

According to [26], we can group the states in Fig. 5 with the same $m_{i}$ (i.e., the states in the same row) together as long as the vertical state-transition rates are much smaller than the horizontal state-transition rates. In DSA, this condition is expected to be met in many applications because spectrum reuse is intended for under-utilized channels with relatively longer ON/OFF periods (e.g., TV bands) compared to the customer arrival/departure by the SUs. In Section VIII, we will quantify the impact of this approximation on the accuracy of the analysis through extensive numerical experiments. 
After the decomposition, the system becomes $M / M / \alpha / \alpha$ while the channel is idle. Hence, we can approximate $\pi_{s_{i}}$ as

$$
\begin{aligned}
& \pi_{s_{i}} \approx \pi_{n_{i} \mid m_{i}} \cdot P\left(m_{i}\right)= \begin{cases}u_{i} & \text { if } s_{i}=(0,0), \\
\pi_{n_{i}} \cdot\left(1-u_{i}\right) & \text { if } m_{i}=1,\end{cases} \\
& \pi_{n_{i}}=\frac{\left(\rho_{i}\right)^{n_{i}} / n_{i} !}{\sum_{n=0}^{\alpha}\left(\rho_{i}\right)^{n} / n !}, \forall n_{i},
\end{aligned}
$$

where $\pi_{n_{i}}$ is the stationary probability of $M / M / \alpha / \alpha$.

Then, revenue and eviction cost occurs only when channel is idle because $n_{i}=0$ for a busy channel. Hence, for $m_{i}=1$, we derive the revenue rate $R_{i}$ (the average revenue per unittime) and the eviction cost $E_{i}$ as follows:

$$
\begin{aligned}
& R_{i}=\sum_{n_{i}=0}^{\alpha} p_{i} n_{i} \cdot \pi_{n_{i}}=p_{i} \rho_{i} \frac{\sum_{n=0}^{\alpha-1}\left(\rho_{i}\right)^{n} / n !}{\sum_{n=0}^{\alpha}\left(\rho_{i}\right)^{n} / n !}, \\
& E_{i}=\sum_{n_{i}=0}^{\alpha} I n_{i} \cdot \pi_{n_{i}} \cdot \Lambda_{i}^{O F F}=\beta \frac{\Lambda_{i}^{O F F}}{\mu} R\left(p_{i}, \rho_{i}\right),
\end{aligned}
$$

where $\Lambda_{i}^{O F F}$ is the transition probability due to the $\mathrm{OFF} \rightarrow \mathrm{ON}$ transition of an idle channel.

The derived $R_{i}$ and $E_{i}$ will become our bases in the later sections to calculate the profit rate of WSP $i$ under various market conditions. For notational convenience, we define

$$
\begin{aligned}
\Delta\left(p_{i}, \Lambda_{i}^{O F F}, \rho_{i}\right) & :=R_{i}-E_{i}=p_{i} \chi\left(\Lambda_{i}^{O F F}, \rho_{i}\right), \\
\chi\left(\Lambda_{i}^{O F F}, \rho_{i}\right) & :=\left(1-\beta \frac{\Lambda_{i}^{O F F}}{\mu}\right) \rho_{i} \frac{\sum_{n=0}^{\alpha-1}\left(\rho_{i}\right)^{n} / n !}{\sum_{n=0}^{\alpha}\left(\rho_{i}\right)^{n} / n !} .
\end{aligned}
$$

Note that it can be seen that $0<\chi\left(\Lambda_{i}^{O F F}, \rho_{i}\right)<1$ by applying the assumptions in Section III.

\section{Analysis of Price Competition Sub-Game}

In this section, we investigate a price competition sub-game to find the best pricing strategy of WSP $i$ in terms of $p_{i}$, when $\left(u_{i}, u_{-i}\right)$ are given. Here, we assume $0 \leq u_{1}, u_{2}<1$ because $u_{-i}=1$ always leads to the price strategy of $p_{i}=\bar{\Pi}$ due to the monopoly of WSP $i .^{9}$

Using the determined strategy, we derive the price NE and the necessary condition for its existence. In addition, we study the impact of price and reimbursement rate on the service preference of customers. Note that the derived price NE will be applied to the quality full-game in Section VI in determining the profit at the equilibrium price for given channel qualities.

\section{A. Achieved Profit by Three Possible Pricing Strategies}

At WSP $i$, the price of the competitor WSP $-i$ (i.e., $p_{-i}$ ) is known since WSP $-i$ advertises its tariff via beacons. Then, WSP $i$ can take one of the following three pricing strategies: (1) $p_{i}<p_{-i}$, (2) $p_{i}>p_{-i}$, and (3) $p_{i}=p_{-i}$. We consider each strategy and derive WSP $i$ 's achieved profit for each case. In addition, we will discuss how to determine the marginal price of WSP $i$ by considering the three cases.

\footnotetext{
${ }^{9}$ Note that $u_{-i}=1$ implies that WSP $-i$ does not provide any service since its channel is always busy.
}

1) Under strategy $1\left(p_{i}<p_{-i}\right)$ : When $p_{i}<p_{-i}$, WSP $i$ 's service is always preferred to its competitor (i.e., $\lambda_{i}=\lambda$ ) since customers choose a cheaper service at their arrival. Hence, WSP $i$ monopolizes the market and it can maximize its profit by setting $p_{i}=p_{-i}-\epsilon, \epsilon>0$, where $\epsilon$ is arbitrarily small. Since $\lambda_{i}=\lambda, \rho_{i}=\rho$ and the profit rate of WSP $i$ becomes

$$
\begin{aligned}
F_{i}^{\left\{p_{i}<p_{-i}\right\}} & =\left(1-u_{i}\right) \cdot \Delta\left(p_{-i}-\epsilon, \Lambda_{i}^{O F F}, \rho\right)-L_{i} \\
& =\left(1-u_{i}\right) \cdot\left(p_{-i}-\epsilon\right) \chi\left(\Lambda_{i}^{O F F}, \rho\right)-L_{i} .
\end{aligned}
$$

2) Under strategy $2\left(p_{i}>p_{-i}\right)$ : When $p_{i}>p_{-i}$, (1) $\lambda_{i}=0$ and $\lambda_{-i}=\lambda$ while WSP $-i$ 's channel is idle, and (2) $\lambda_{i}=\lambda$ and $\lambda_{-i}=0$ while WSP $-i$ 's channel is busy and WSP $i$ 's channel is idle. That is, even though WSP $i$ provides a more expensive service, customers will still choose WSP $i$ if WSP $-i$ 's channel is busy. In such a case, WSP $i$ can maximize its profit by setting $p_{i}=\bar{\Pi}$. Therefore, with probability $\left(1-u_{i}\right) u_{-i}$ the WSP $i$ 's system takes stationary probability of Eq. (2), and since $\rho_{i}=\rho$ its profit rate becomes

$$
\begin{aligned}
F_{i}^{\left\{p_{i}>p_{-i}\right\}} & =\left(1-u_{i}\right) u_{-i} \cdot \Delta\left(\bar{\Pi}, \Lambda_{i}^{O F F}, \rho\right)-L_{i} \\
& =\left(1-u_{i}\right) u_{-i} \cdot \bar{\Pi} \chi\left(\Lambda_{i}^{O F F}, \rho\right)-L_{i} .
\end{aligned}
$$

3) Under strategy $3\left(p_{i}=p_{-i}\right)$ : For $p_{i}=p_{-i}$, two cases are considered. First, when $m_{i}=m_{-i}=1$, two WSPs take an equal share of the market such that $\lambda_{i}=\lambda_{-i}=\lambda / 2$ (i.e., $\rho_{i}=\rho_{-i}=\rho / 2$ ). Second, when $m_{i}=1$ and $m_{-i}=$ 0 , we have $\lambda_{i}=\lambda$ (i.e., $\rho_{i}=\rho$ ) and $\lambda_{-i}=0$ since the arriving customers cannot find the service beacons of WSP $-i$. Therefore, by setting $p_{i}=p_{-i}$, the profit rate of WSP $i$ becomes

$$
\begin{aligned}
F_{i}^{\left\{p_{i}=p_{-i}\right\}} & =\left(1-u_{i}\right)\left\{u_{-i} \cdot \Delta\left(p_{-i}, \Lambda_{i}^{O F F}, \rho\right)\right. \\
& \left.+\left(1-u_{-i}\right) \cdot \Delta\left(p_{-i}, \Lambda_{i}^{O F F}, \rho / 2\right)\right\}-L_{i} \\
& =\left(1-u_{i}\right)\left\{u_{-i} \cdot p_{-i} \chi\left(\Lambda_{i}^{O F F}, \rho\right)\right. \\
& \left.+\left(1-u_{-i}\right) \cdot p_{-i} \chi\left(\Lambda_{i}^{O F F}, \rho / 2\right)\right\}-L_{i} .
\end{aligned}
$$

4) Determination of the Marginal Price: We define the marginal price $\underline{\Pi}_{i}$ as the minimal price to guarantee a nonnegative profit for WSP $i$ even at the worst case. That is, when $p_{i}=\underline{\Pi}_{i}$, the WSP $i$ should be able to achieve at least a break-even (zero profit) regardless of $p_{-i}$.

When we fix $p_{i}=\underline{\Pi}_{i}$, the profit rate $F_{i}$ previously given as Eqs. (5), (6), (7) becomes

$$
\begin{aligned}
F_{i}^{\left\{p_{i}<p_{-i}\right\}}= & \left(1-u_{i}\right) \cdot \Delta\left(\underline{\Pi}_{i}, \Lambda_{i}^{O F F}, \rho\right)-L_{i}, \\
F_{i}^{\left\{p_{i}>p_{-i}\right\}}= & \left(1-u_{i}\right) \cdot u_{-i} \Delta\left(\underline{\Pi}_{i}, \Lambda_{i}^{O F F}, \rho\right)-L_{i}, \\
F_{i}^{\left\{p_{i}=p_{-i}\right\}}= & \left(1-u_{i}\right)\left\{u_{-i} \Delta\left(\underline{\Pi}_{i}, \Lambda_{i}^{O F F}, \rho\right)\right. \\
& \left.+\left(1-u_{-i}\right) \Delta\left(\underline{\Pi}_{i}, \Lambda_{i}^{O F F}, \rho / 2\right)\right\}-L_{i} .
\end{aligned}
$$

One can observe that $F_{i}^{\left\{p_{i}>p_{-i}\right\}}$ is the worst. Therefore, to guarantee $F_{i} \geq 0$, we need to set

$$
\underline{\Pi}_{i}=\frac{L_{i} /\left(1-u_{i}\right)}{u_{-i} \chi\left(\Lambda_{i}^{O F F}, \rho\right)}=\frac{\bar{\gamma}_{1}\left(2-u_{i}-u_{-i}\right)^{\gamma_{2}}}{u_{-i \chi}\left(\Lambda_{i}^{O F F}, \rho\right)},
$$

where Eqs. (1) and (3) are applied.

Note that if Eq. (8) yields $\underline{\Pi}_{i}>\bar{\Pi}$, WSP $i$ cannot make a positive profit for any $p_{i}$ since $\lambda_{i}=0$ for $p_{i}>\bar{\Pi}$ making the 
profit strictly negative such as $F_{i}=-L_{i}$. Therefore, WSP $i$ should opt out of the market when $\underline{\Pi}_{i}>\bar{\Pi}$ in order not to incur any channel leasing cost. In the next section, this will be modeled as forcing $u_{i}=1$ at the quality competition where the channel leasing cost becomes zero since $C_{i}^{\text {eff }}=0$.

\section{B. Optimal Pricing Strategy}

The goal of WSP $i$ is to maximize its profit by optimally determining its price $p_{i}$ for a given $p_{-i}$, for which the profit rates of the three pricing strategies in Section V-A are compared as follows.

Case 1 (if $\left.p_{-i}<\underline{\Pi}_{i}\right)$ : strategy $2\left(p_{i}=\bar{\Pi}>p_{-i}\right)$ is the only possible strategy, since other two strategies violate $p_{i} \geq \underline{\Pi}_{i}$.

Case 2 (if $p_{-i}=\underline{\Pi}_{i}$ ): the optimal strategy is either strategy 2 $\left(p_{i}=\bar{\Pi}>p_{-i}\right)$ or strategy $3\left(p_{i}=p_{-i}=\underline{\Pi}_{i}\right)$, since strategy 1 violates $p_{i} \geq \underline{\Pi}_{i}$.

Case 3 (if $\Pi_{i}<p_{-i}<\bar{\Pi}$ ): the optimal strategy is either strategy $1\left(p_{i}=p_{-i}-\epsilon<p_{-i}\right)$, strategy $2\left(p_{i}=\bar{\Pi}>p_{-i}\right)$, or strategy $3\left(p_{i}=p_{-i}\right)$.

Case 4 (if $p_{-i}=\bar{\Pi}$ ): the optimal strategy is either strategy $1\left(p_{i}=p_{-i}-\epsilon<p_{-i}\right)$ or strategy $3\left(p_{i}=\bar{\Pi}=p_{-i}\right)$, since strategy 2 violates $p_{i} \leq \bar{\Pi}$.

We first consider the difference of profit rates between strategies 1 and 3 according to Eqs. (5) and (7):

$$
\begin{aligned}
& F_{i}^{\left\{p_{i}<p_{-i}\right\}}-F_{i}^{\left\{p_{i}=p_{-i}\right\}}=\left(1-u_{i}\right)\left(1-u_{-i}\right) p_{-i} \times \\
& \left\{\chi\left(\Lambda_{i}^{O F F}, \rho\right)-\chi\left(\Lambda_{i}^{O F F}, \rho / 2\right)\right\}-\epsilon\left(1-u_{i}\right) \chi\left(\Lambda_{i}^{O F F}, \rho\right)
\end{aligned}
$$

from which we derive Lemma 1 as follows.

Lemma 1. Suppose $\underline{\Pi}_{i}<p_{-i} \leq \bar{\Pi}$. Then, for WSP $i$, price strategy $3\left(p_{i}=p_{-i}\right)$ is strictly dominated by price strategy 1 $\left(p_{i}=p_{-i}-\epsilon<p_{-i}\right)$ with an arbitrarily small $\epsilon>0$.

Proof: In Eq. (9), $F_{i}^{\left\{p_{i}<p_{-i}\right\}}>F_{i}^{\left\{p_{i}=p_{-i}\right\}}$ for an arbitrarily small $\epsilon$, since it can be shown that

$$
\begin{gathered}
\chi\left(\Lambda_{i}^{O F F}, \rho\right)-\chi\left(\Lambda_{i}^{O F F}, \rho / 2\right)=\left(1-\beta \frac{\Lambda_{i}^{O F F}}{\mu}\right) \times \\
\rho\left\{\frac{\sum_{n=0}^{\alpha-1} \rho^{n} / n !}{\sum_{n=0}^{\alpha} \rho^{n} / n !}-\frac{1}{2} \cdot \frac{\sum_{n=0}^{\alpha-1}(\rho / 2)^{n} / n !}{\sum_{n=0}^{\alpha}(\rho / 2)^{n} / n !}\right\}>0
\end{gathered}
$$

as follows. First, $\left(1-\beta \Lambda_{i}^{O F F} / \mu\right)>0$ because $\beta \leq 1$ and $\Lambda_{i}^{O F F} / \mu<1$ by our assumption. Second,

$$
\begin{aligned}
& \frac{\sum_{n=0}^{\alpha} \rho^{n} / n !}{\sum_{n=0}^{\alpha-1} \rho^{n} / n !}-2 \cdot \frac{\sum_{n=0}^{\alpha}(\rho / 2)^{n} / n !}{\sum_{n=0}^{\alpha-1}(\rho / 2)^{n} / n !}= \\
& \quad-1+\frac{\rho^{\alpha} / \alpha !}{\sum_{n=0}^{\alpha-1} \rho^{n} / n !}-\frac{2 \cdot(\rho / 2)^{\alpha} / \alpha !}{\sum_{n=0}^{\alpha-1}(\rho / 2)^{n} / n !}<0,
\end{aligned}
$$

where $\frac{\rho^{\alpha} / \alpha !}{\sum_{n=0}^{\alpha-1} \rho^{n} / n !}<1$ because $\rho^{\alpha} / \alpha$ ! $<1$ for $0<\rho<1$, $\alpha \geq 1$, and $\sum_{n=0}^{\alpha-1} \rho^{n} / n !=1+\sum_{n=1}^{\alpha-1} \rho^{n} / n !>1$.

Next, we consider the difference of profit rates between strategies 1 and 2 according to Eqs. (5) and (6):

$$
\begin{aligned}
F_{i}^{\left\{p_{i}<p_{-i}\right\}} & -F_{i}^{\left\{p_{i}>p_{-i}\right\}} \\
& =\left(1-u_{i}\right)\left(p_{-i}-\epsilon-u_{-i} \bar{\Pi}\right) \chi\left(\Lambda_{i}^{O F F}, \rho\right)
\end{aligned}
$$

from which we derive Lemma 2 as follows.

Lemma 2. Suppose $\underline{\Pi}_{i}<p_{-i}<\bar{\Pi}$. Then, for WSP $i$,
- Strategy $1\left(p_{i}=p_{-i}-\epsilon\right)$ dominates strategy $2\left(p_{i}=\bar{\Pi}\right)$, when $p_{-i}>u_{-i} \cdot \bar{\Pi}$, and

- Strategy $2\left(p_{i}=\bar{\Pi}\right)$ dominates strategy $1\left(p_{i}=p_{-i}-\epsilon\right)$, when $p_{-i} \leq u_{-i} \cdot \bar{\Pi}$,

with an arbitrarily small $\epsilon>0$.

Proof: The lemma directly follows from Eq. (10).

We also consider the difference of profit rates between strategies 2 and 3 provided that $p_{-i}=\underline{\Pi}_{i}$ (i.e., Case 2), using Eqs. (6) and (7):

$$
\begin{aligned}
F_{i}^{\left\{p_{i}>p_{-i}\right\}}-F_{i}^{\left\{p_{i}=p_{-i}\right\}} & =\left(1-u_{i}\right)\left\{u_{-i}\left(\bar{\Pi}-\underline{\Pi}_{i}\right) \times\right. \\
\chi\left(\Lambda_{i}^{O F F}, \rho\right) & \left.-\left(1-u_{-i}\right) \underline{\Pi}_{i} \chi\left(\Lambda_{i}^{O F F}, \rho / 2\right)\right\}
\end{aligned}
$$

from which we derive Lemma 3 as follows.

Lemma 3. Suppose $p_{-i}=\underline{\Pi}_{i}$. Then, for WSP $i$,

- Strategy $2\left(p_{i}=\bar{\Pi}\right)$ dominates strategy $3\left(p_{i}=\underline{\Pi}_{i}\right)$, when $\frac{u_{-i}}{1-u_{-i}}\left(\frac{\bar{\Pi}}{\underline{\Pi}_{i}}-1\right)>\frac{\chi\left(\Lambda_{i}^{O F F}, \rho / 2\right)}{\chi\left(\Lambda_{i}^{O F F}, \rho\right)}$, and

- Strategy $3\left(p_{i}=\underline{\Pi}_{i}\right)$ dominates strategy $2\left(p_{i}=\bar{\Pi}\right)$, when $\frac{u_{-i}}{1-u_{-i}}\left(\frac{\bar{\Pi}_{i}}{\underline{\Pi}_{i}}-1\right) \leq \frac{\chi\left(\Lambda_{i}^{O F F}, \rho / 2\right)}{\chi\left(\Lambda_{i}^{O F F}, \rho\right)}$.

Proof: The lemma directly follows from Eq. (11). Finally, Theorem 1 derives the best response $p_{i}\left(p_{-i}\right)$.

Theorem 1. The best response function $p_{i}\left(p_{-i}\right)$ is

- When $\frac{u_{-i}}{1-u_{-i}}\left(\frac{\bar{\Pi}}{\underline{\Pi}_{i}}-1\right)>\frac{\chi\left(\Lambda_{i}^{O F F}, \rho / 2\right)}{\chi\left(\Lambda_{i}^{O F F}, \rho\right)}$ : $p_{i}\left(p_{-i}\right)=\left\{\begin{array}{l}\bar{\Pi}, \quad \text { when } \underline{\Pi}_{-i} \leq p_{-i} \leq \max \left\{u_{-i} \bar{\Pi}, \underline{\Pi}_{i}\right\}, \\ p_{-i}-\epsilon, \text { when } \max \left\{u_{-i} \bar{\Pi}, \underline{\Pi}_{i}\right\}<p_{-i} \leq \bar{\Pi} .\end{array}\right.$

- When $\frac{u_{-i}}{1-u_{-i}}\left(\frac{\bar{\Pi}}{\underline{\Pi}_{i}}-1\right) \leq \frac{\chi\left(\Lambda_{i}^{O F F}, \rho / 2\right)}{\chi\left(\Lambda_{i}^{O F F}, \rho\right)}$ :

$$
p_{i}\left(p_{-i}\right)= \begin{cases}\bar{\Pi}_{i}, & \text { when } \underline{\Pi}_{-i} \leq p_{-i} \leq \underline{\Pi}_{i}, \\ \underline{\Pi}_{i}, & \text { when } p_{-i}=\underline{\Pi}_{i}, \\ p_{-i}-\epsilon, & \text { when } \underline{\Pi}_{i}<p_{-i} \leq \bar{\Pi} .\end{cases}
$$

Proof: The theorem is derived by applying Lemmas 1, 2, and 3 to Cases $1-4$.

\section{Nash Equilibrium of the Price Competition}

Based on the derived optimal price strategy in Theorem 1, we can find the Nash Equilibrium (NE) of the price competition. To find the NE, we consider the case where $\underline{\Pi}_{i} \leq \bar{\Pi}$ and $\underline{\Pi}_{-i} \leq \bar{\Pi}$ so that both WSPs may participate in the market competition. A price NE $\left(p_{1}, p_{2}\right)=\left(p_{1}^{*}, p_{2}^{*}\right)$, if it exists, should satisfy: $p_{1}^{*}=p_{1}\left(p_{2}^{*}\right)$ and $p_{2}^{*}=p_{2}\left(p_{1}^{*}\right)$. Using this fact and Theorem 1, we first derive Lemma 4 and then determine the price NE in Theorem 2.

Lemma 4. There exists no price $N E$ if $\frac{u_{-i}}{1-u_{-i}}\left(\frac{\bar{\Pi}}{\underline{\Pi}_{i}}-1\right)>$ $\frac{\chi\left(\Lambda_{i}^{O F F}, \rho / 2\right)}{\chi\left(\Lambda_{i}^{O F F}, \rho\right)}$ for any $i \in\{1,2\}$.

Proof: Without loss of generality, let us assume $i=1$. For WSP 1, there are two possible strategies: $p_{1}\left(p_{2}\right)=\bar{\Pi}$ or $p_{1}\left(p_{2}\right)=p_{2}-\epsilon$. First, if $p_{1}^{*}=p_{1}\left(p_{2}^{*}\right)=\bar{\Pi}, p_{2}^{*}$ must satisfy $\underline{\Pi}_{2} \leq p_{2}^{*} \leq \max \left\{u_{2} \bar{\Pi}, \underline{\Pi}_{1}\right\}$. At NE, $p_{1}^{*}$ and $p_{2}^{*}$ must also satisfy $p_{2}^{*}=p_{2}\left(p_{1}^{*}\right)=p_{2}(\bar{\Pi})=\bar{\Pi}-\epsilon$ since $p_{1}^{*}=\bar{\Pi}$. This implies that $\underline{\Pi}_{2} \leq \bar{\Pi}-\epsilon \leq \max \left\{u_{2} \bar{\Pi}, \underline{\Pi}_{1}\right\}$ for an arbitrarily small $\epsilon>0$ which is a contradiction since $u_{2}<1$ by assumption. Therefore, none of $p_{1}^{*}$ and $p_{2}^{*}$ can be equal to $\bar{\Pi}$ at the price NE. Second, $p_{1}^{*}=p_{1}\left(p_{2}^{*}\right)=p_{2}^{*}-\epsilon$ 
and $p_{2}^{*}=p_{2}\left(p_{1}^{*}\right)=\underline{\Pi}_{2}$ may not happen because the latter implies $p_{1}^{*}=\underline{\Pi}_{2}$ while the former implies $p_{1}^{*}=\underline{\Pi}_{2}-\epsilon$, which is a contradiction. Finally, $p_{1}^{*}=p_{1}\left(p_{2}^{*}\right)=p_{2}^{*}-\epsilon$ and $p_{2}^{*}=p_{2}\left(p_{1}^{*}\right)=p_{1}^{*}-\epsilon$ lead to $p_{1}^{*}=p_{1}^{*}-2 \epsilon$ which is a contradiction since $\epsilon>0$. As a result, there exists no price NE when $\frac{u_{-i}}{1-u_{-i}}\left(\frac{\bar{\Pi}}{\underline{\Pi}_{i}}-1\right)>\frac{\chi\left(\Lambda_{i}^{O F F}, \rho / 2\right)}{\chi\left(\Lambda_{i}^{O F F}, \rho\right)}$ for any $i$.

Finally, Theorem 2 determines the price NE.

Theorem 2. Price NE exists only when $\underline{\Pi}_{1}=\underline{\Pi}_{2}$ and $\frac{u_{-i}}{1-u_{-i}}\left(\frac{\bar{\Pi}_{\underline{I}}}{\underline{\Pi}_{i}}-1\right) \leq \frac{\chi\left(\Lambda_{i}^{O F F}, \rho / 2\right)}{\chi\left(\Lambda_{i}^{O F F}, \rho\right)}, \forall i$, under which a unique price $N E$ is determined as $\left(p_{1}^{*}, p_{2}^{*}\right)=(\underline{\Pi}, \underline{\Pi}), \underline{\Pi}:=\underline{\Pi}_{1}=\underline{\Pi}_{2}$.

Proof: There are three possibilities such as $p_{i}\left(p_{-i}\right)=\bar{\Pi}$, $\underline{\Pi}_{i}$, or $p_{-i}-\epsilon$. As previously shown in the proof of Lemma 4 , however, $p_{i}^{*}$ cannot be equal to $\bar{\Pi}$ or $p_{-i}-\epsilon$ at a price NE. Then, only possibility is $p_{1}^{*}=p_{1}\left(p_{2}^{*}\right)=\underline{\Pi}_{1}$ and $p_{2}^{*}=p_{2}\left(p_{1}^{*}\right)=\underline{\Pi}_{2}$, provided that $p_{1}^{*}=\underline{\Pi}_{2}$ and $p_{2}^{*}=\underline{\Pi}_{1}$. It can be seen that this condition holds only when $\underline{\Pi}_{1}=\underline{\Pi}_{2}$. Therefore, a price NE exists at $\left(p_{1}^{*}, p_{2}^{*}\right)=\left(\underline{\Pi}_{1}, \underline{\Pi}_{2}\right)$ when $\underline{\Pi}_{1}=\underline{\Pi}_{2}$, where $p_{1}^{*}=p_{2}^{*}$. This completes the proof.

\section{Achievability of Price NE}

In Section V-A, WSP $i$ sets its price according to the competitor WSP $-i$ 's price which is advertised via WSP $-i$ 's beacons. Then, the question arises when WSP $i$ never received beacons from WSP $-i$ and has to set its price $p_{i}$. In such a case, the concept of NE implies that WSP $i$ should set its price as $p_{i}=\underline{\Pi}$ (provided that the NE existence condition is met) as directed in Theorem 2 since it ensures the price NE will be achieved. Specifically, once WSP $i$ 's price is advertised as $p_{i}=\underline{\Pi}$ and heard by WSP $-i$, WSP $-i$ will set its price as $p_{-i}=\underline{\Pi}$ which leads to the price NE.

\section{Analysis of Quality Competition Full-Game}

The goal of the quality competition is to find the best channel to lease with the optimal quality $\left(u_{i}, \Lambda_{i}^{O F F}\right)$ that achieves the maximal profit of WSP $i$ at the equilibrium price found in Section V. In terms of the best response function, this implies that an optimal pair of $\left(u_{i}, \Lambda_{i}^{O F F}\right)$ should be determined for each given pair of $\left(u_{-i}, \Lambda_{-i}^{O F F}\right)$. However, it will be shown in Section VI-A that there exists a one-to-one relationship between the optimal $u_{i}$ and $\Lambda_{i}^{O F F}$, and thus we can consider the best response function for either $u_{i}$ or $\Lambda_{i}^{O F F}$.

Note that in this section $\gamma_{2}=1$ is considered as an illustrative example. However, the same procedure is applicable for any $\gamma_{2}$ as it only affects how the interval of $\Lambda_{i}^{O F F}$ is determined in Section VI-B.

\section{A. Two Constraints on the Existence of Price NE}

In Theorem 2, we have shown that there exist two necessary conditions for the existence of price NE, such as:

Condition I: $\underline{\Pi}=\underline{\Pi}_{i}=\underline{\Pi}_{-i}$,

Condition II: $\frac{u_{-i}}{1-u_{-i}}\left(\frac{\bar{\Pi}}{\underline{\Pi}_{i}}-1\right) \leq \frac{\chi\left(\Lambda_{i}^{O F F}, \rho / 2\right)}{\chi\left(\Lambda_{i}^{O F F}, \rho\right)}, \forall i$.
Eq. (12) can be further simplified by applying Eq. (4):

$$
\begin{aligned}
\frac{\bar{\gamma}_{1}\left(2-u_{i}-u_{-i}\right)^{\gamma_{2}}}{u_{-i} \chi\left(\Lambda_{i}^{O F F}, \rho\right)} & =\frac{\bar{\gamma}_{1}\left(2-u_{i}-u_{-i}\right)^{\gamma_{2}}}{u_{i} \chi\left(\Lambda_{-i}^{O F F}, \rho\right)} \\
\Rightarrow \quad u_{i} & =\left(\frac{1-\frac{\beta}{\mu} \Lambda_{i}^{O F F}}{1-\frac{\beta}{\mu} \Lambda_{-i}^{O F F}}\right) u_{-i}
\end{aligned}
$$

from which it is seen that there exists a one-to-one relationship between the optimal $u_{i}$ and $\Lambda_{i}^{O F F}$, for given $\left(u_{-i}, \Lambda_{-i}^{O F F}\right)$. Therefore, we can consider the best response function for either $u_{i}$ or $\Lambda_{i}^{O F F}$, e.g., $u_{i}\left(u_{-i}\right)$ or $\Lambda_{i}^{O F F}\left(\Lambda_{-i}^{O F F}\right)$.

Considering $0 \leq u_{i}<1$, an interval of $\Lambda_{i}^{O F F}$ can be obtained from Eq. (14) such that

$$
\frac{\mu}{\beta}\left\{1-\left(1-\frac{\beta}{\mu} \Lambda_{-i}^{O F F}\right) \cdot \frac{1}{u_{-i}}\right\}<\Lambda_{i}^{O F F} \leq \frac{\mu}{\beta} .
$$

\section{B. Market Entry Barrier}

WSP $i$ 's profit becomes strictly negative in case its marginal price becomes greater than the monopoly price (i.e., $\underline{\Pi}_{i}>\bar{\Pi}$ ), because there will be no customer arrival while the channel leasing fee must be still paid. If this happens, the WSP would rather shut down its service by not leasing any channel (equivalently, leasing a channel with $u_{i}=1$ ). Therefore, there exists the market entry condition for a WSP, which is described by $\underline{\Pi}_{i} \leq \bar{\Pi}$. For a given $u_{-i}$, this condition results in the following interval of $u_{i}$ :

$$
\begin{aligned}
\underline{\Pi}_{i} & =\frac{\bar{\gamma}_{1}\left(2-u_{i}-u_{-i}\right)^{\gamma_{2}}}{u_{-i} \chi\left(\Lambda_{i}^{O F F}, \rho\right)} \leq \bar{\Pi} \\
& \Rightarrow u_{i} \geq 2-u_{-i}-\left\{\frac{\bar{\Pi} \chi\left(\Lambda_{i}^{O F F}, \rho\right)}{\bar{\gamma}_{1}} \cdot u_{-i}\right\}^{1 / \gamma_{2}}, \forall i .
\end{aligned}
$$

Using Eq. (14), Eq. (16) can be transformed into a function of $\Lambda_{i}^{O F F}$ such as

$$
\begin{aligned}
& 2-u_{-i} \leq \frac{u_{-i}}{\left(1-\frac{\beta}{\mu} \Lambda_{-i}^{O F F}\right)} \cdot\left(1-\frac{\beta}{\mu} \Lambda_{i}^{O F F}\right) \\
& +\left\{\frac{\bar{\Pi}}{\bar{\gamma}_{1}} \cdot \frac{u_{-i} \chi\left(\Lambda_{-i}^{O F F}, \rho\right)}{\left(1-\frac{\beta}{\mu} \Lambda_{-i}^{O F F}\right)}\right\}^{1 / \gamma_{2}} \cdot\left(1-\frac{\beta}{\mu} \Lambda_{i}^{O F F}\right)^{1 / \gamma_{2}}
\end{aligned}
$$

from which another interval of $\Lambda_{i}^{O F F}$ can be obtained. In case $\gamma_{2}=1$, Eq. (17) produces

$$
\Lambda_{i}^{O F F} \leq \frac{\mu}{\beta}\left\{1-\frac{2-u_{-i}}{u_{-i}} \cdot \frac{\left(1-\frac{\beta}{\mu} \Lambda_{-i}^{O F F}\right)}{1+\left(\bar{\Pi} / \bar{\gamma}_{1}\right) \chi\left(\Lambda_{-i}^{O F F}, \rho\right)}\right\}
$$

where the equality holds when $\underline{\Pi}_{i}=\bar{\Pi}$.

\section{Optimal Quality Strategy}

For WSP $i$, the objective of quality strategy is to determine the optimal $u_{i}$ (or $\Lambda_{i}^{O F F}$ ) for given $\left(u_{-i}, \Lambda_{-i}^{O F F}\right)$ to maximize its profit measured at the price NE $(\underline{\Pi}, \underline{\Pi})$. Since $p_{i}=p_{-i}$ at the NE of the price competition, the resulting profit of WSP $i$ is given as Eq. (7) and the best response function $u_{i}\left(u_{-i}, \Lambda_{-i}^{O F F}\right)$ to maximize such a profit can be determined 
as

$$
\begin{aligned}
u_{i}^{*}= & u_{i}\left(u_{-i}, \Lambda_{-i}^{O F F}\right)=\operatorname{argmax}_{0 \leq u_{i}<1} F_{i}^{\left\{p_{i}=p_{-i}\right\}}, \\
& \text { where } p_{i}=p_{-i}=\underline{\Pi}_{i}=\underline{\Pi}_{-i}=\underline{\Pi}, \\
& \text { subject to Eqs. (12), (13), and (16). }
\end{aligned}
$$

Note that in case no $u_{i}$ exists that satisfies Eq. (19), we set $u_{i}^{*}=1$ (i.e., no service).

By combining Eqs. (7) and (8), WSP $i$ 's profit is derived as

$$
\begin{aligned}
& F_{i}\left\{p_{i}=p_{-i}\right\} \\
&=\left(1-u_{i}\right) \underline{\Pi}\left\{u_{-i \chi}\left(\Lambda_{i}^{O F F}, \rho\right)+\left(1-u_{-i}\right) \chi\left(\Lambda_{i}^{O F F}, \rho / 2\right)\right\} \\
& \quad-\left(1-u_{i}\right) \underline{\Pi} u_{-i \chi} \chi\left(\Lambda_{i}^{O F F}, \rho\right) \\
&=\bar{\gamma}_{1} \frac{\chi\left(\Lambda_{i}^{O F F}, \rho / 2\right)}{\chi\left(\Lambda_{i}^{O F F}, \rho\right)}\left(1-\Psi_{-i}+\frac{\beta}{\mu} \Psi_{-i} \Lambda_{i}^{O F F}\right) \times \\
&\left(2-u_{-i}-\Psi_{-i}+\frac{\beta}{\mu} \Psi_{-i} \Lambda_{i}^{O F F}\right)^{\gamma_{2}} \frac{\left(1-u_{-i}\right)}{u_{-i}}
\end{aligned}
$$

where Eqs. (8) and (14) are applied, and

$$
u_{i}=\Psi_{-i}-\left(\frac{\beta}{\mu} \Psi_{-i}\right) \Lambda_{i}^{O F F}, \quad \Psi_{-i}:=\frac{u_{-i}}{\left(1-\frac{\beta}{\mu} \Lambda_{-i}^{O F F}\right)} .
$$

Using Eq. (20), Eq. (19) can be re-written for $\gamma_{2}=1$ as

$$
\begin{array}{r}
\Lambda_{i}^{O F F *}=\underset{\Lambda_{i}^{O F F}>0}{\operatorname{argmax}}\left\{\left(1-\Psi_{-i}+(\beta / \mu) \Psi_{-i} \Lambda_{i}^{O F F}\right)\right. \\
\left.\left(2-u_{-i}-\Psi_{-i}+(\beta / \mu) \Psi_{-i} \Lambda_{i}^{O F F}\right)\right\},
\end{array}
$$

subject to Eqs. (15), (13), and (18)

where two constraints on $u_{i}$, i.e., Eqs. (12) and (16), are replaced with the constraints on $\Lambda_{i}^{O F F}$, i.e., Eqs. (15) and (18), and the terms in Eq. (20) not relevant to $\Lambda_{i}^{O F F}$ are ignored. Note that $\frac{\chi\left(\Lambda_{i}^{O F F}, \rho / 2\right)}{\chi\left(\Lambda_{i}^{O F F}, \rho\right)}$ only depends on $\rho$, not on $\Lambda_{i}^{O F F}$.

Combining the two intervals in Eqs. (15) and (18) yields

$$
\begin{aligned}
& \frac{\mu}{\beta}\left\{1-\left(1-\frac{\beta}{\mu} \Lambda_{-i}^{O F F}\right) \frac{1}{u_{-i}}\right\}<\Lambda_{i}^{O F F} \leq \\
& \frac{\mu}{\beta}\left\{1-\frac{2-u_{-i}}{u_{-i}} \cdot \frac{\left(1-\frac{\beta}{\mu} \Lambda_{-i}^{O F F}\right)}{1+\left(\bar{\Pi} / \bar{\gamma}_{1}\right) \chi\left(\Lambda_{-i}^{O F F}, \rho\right)}\right\},
\end{aligned}
$$

provided that in Eq. (22) the upperbound is greater than the lowerbound, which equals to the condition:

$$
\frac{2-u_{-i}}{1+\left(\bar{\Pi} / \bar{\gamma}_{1}\right) \chi\left(\Lambda_{-i}^{O F F}, \rho\right)}<1
$$

Here, our approach is as follows. First, we try to find $\Lambda_{i}^{O F F *}$ in Eq. (21) by considering only two constraints: Eqs. (15) and (18), or equivalently Eq. (22). Then, we check if the derived $\Lambda_{i}^{O F F *}$ satisfies the remaining constraint Eq. (13) and the necessary condition Eq. (23). Based on the approach, the best response functions are obtained as in Theorem 3. and $u_{i}\left(u_{-i}, \Lambda_{-i}^{O F F}\right)$ are determined as

$$
\begin{aligned}
\Lambda_{i}^{O F F *} & =\Lambda_{i}^{O F F}\left(u_{-i}, \Lambda_{-i}^{O F F}\right) \\
& =\frac{\mu}{\beta}\left\{1-\frac{\left(\left(2-u_{-i}\right) / u_{-i}\right)\left(1-\frac{\beta}{\mu} \Lambda_{-i}^{O F F}\right)}{1+\left(\bar{\Pi} / \bar{\gamma}_{1}\right) \chi\left(\Lambda_{-i}^{O F F}, \rho\right)}\right\}, \\
u_{i}^{*} & =u_{i}\left(u_{-i}, \Lambda_{-i}^{O F F}\right)=\frac{2-u_{-i}}{1+\left(\bar{\Pi} / \bar{\gamma}_{1}\right) \chi\left(\Lambda_{-i}^{O F F}, \rho\right)} .
\end{aligned}
$$

Proof: Since $f\left(\Lambda_{i}^{O F F}\right):=\left(1-\Psi_{-i}+\right.$ $\left.(\beta / \mu) \Psi_{-i} \Lambda_{i}^{O F F}\right)\left(2-u_{-i}-\Psi_{-i}+(\beta / \mu) \Psi_{-i} \Lambda_{i}^{O F F}\right)$ is a convex function of $\Lambda_{i}^{O F F}$, its maximum exists at either of the two extreme values of $\Lambda_{i}^{O F F}$ given by Eq. (22). It is trivial to show that $f\left(\Lambda_{i}^{O F F}\right)$ is maximized at the upperbound of $\Lambda_{i}^{O F F}$, thus obtaining Eq. (24). Then, Eq. (25) is derived by applying Eq. (24) to Eq. (14).

Corollary 1. At the maximal profit achieved by $\Lambda_{i}^{O F F *}$ and $u_{i}^{*}$ in Theorem 3, we have $\underline{\Pi}_{i}=\bar{\Pi}$.

Proof: Since $\left(u_{1}^{*}, \Lambda_{1}^{O F F *}\right)$ in Theorem 3 results from the equality condition in Eq. (18), $\underline{\Pi}_{i}=\bar{\Pi}$.

Corollary 2. The best response functions in Theorem 3 satisfy the two necessary conditions Eq. (13) and Eq. (23).

Proof: By Corollary $1, \underline{\Pi}_{i}=\bar{\Pi}$ is achieved by the best response functions. Therefore, Eq. (13) holds because the lefthand side of Eq. (13) becomes zero while its right-hand side is positive. Next, the form of Eq. (25) is the same as the lefthand side of Eq. (23), and thus Eq. (23) holds since $u_{1}^{*}<1$.

\section{Nash Equilibrium of the Quality Competition}

A quality $\mathrm{NE}\left(u_{1}, u_{2}\right)=\left(u_{1}^{*}, u_{2}^{*}\right)$, or equivalently $\left(\Lambda_{1}^{O F F}, \Lambda_{2}^{O F F}\right)=\left(\Lambda_{1}^{O F F *}, \Lambda_{2}^{O F F *}\right)$, should satisfy:

$\left\{\begin{array}{l}u_{1}^{*}=u_{1}\left(u_{2}^{*}, \Lambda_{2}^{O F F *}\right) \\ u_{2}^{*}=u_{2}\left(u_{1}^{*}, \Lambda_{1}^{O F F *}\right)\end{array}\right.$ or $\left\{\begin{array}{l}\Lambda_{1}^{O F F *}=\Lambda_{1}^{O F F}\left(u_{2}^{*}, \Lambda_{2}^{O F F *}\right) \\ \Lambda_{2}^{O F F *}=\Lambda_{2}^{O F F}\left(u_{1}^{*}, \Lambda_{1}^{O F F *}\right)\end{array}\right.$

By combining Eq. (26) with Eq. (25) in Theorem 3, the quality $\mathrm{NE}\left(u_{1}^{*}, u_{2}^{*}\right)$ is determined as

$$
\begin{aligned}
& u_{1}^{*}=\frac{2\left(\hat{\chi}_{1}-1\right)}{\hat{\chi}_{1} \hat{\chi}_{2}-1}, \quad u_{2}^{*}=\frac{2\left(\hat{\chi}_{2}-1\right)}{\hat{\chi}_{1} \hat{\chi}_{2}-1}, \\
& \hat{\chi}_{i}:=1+\frac{\bar{\Pi}}{\bar{\gamma}_{1}} \chi\left(\Lambda_{i}^{O F F^{*}}, \rho\right) .
\end{aligned}
$$

Since $\hat{\chi}_{i}$ is solely determined by $\Lambda_{i}^{O F F *}$ (using the given set of constants $\bar{\Pi}, \rho$, and $\bar{\gamma}_{1}$ ), a quality NE is uniquely determined as Eq. (27) for given $\left(\Lambda_{1}^{O F F}, \Lambda_{2}^{O F F}\right)$.

There exist, however, vastly many possible $\left(\Lambda_{1}^{O F F}, \Lambda_{2}^{O F F}\right)$, and hence Eq. (27) itself does not represent a unique NE. ${ }^{10}$ According to the concept of focal point [27], WSPs 1 and 2 may agree on an equilibrium $\left(\Lambda_{1}^{O F F *}, \Lambda_{2}^{O F F *}\right)$ that maximizes their profit on a fair basis, i.e., achieving the same profit. 
Using Eqs. (27) and (28), the profit of WSP $i$ in Eq. (20) can be expressed in terms of $\hat{\chi}_{i}$ such as

$$
\begin{aligned}
F_{i}^{\left\{p_{i}=p_{-i}\right\}=} & \frac{1}{\bar{\gamma}_{1}} \cdot \frac{\chi\left(\Lambda_{i}^{O F F}, \rho\right)}{\chi\left(\Lambda_{i}^{O F F}, \rho / 2\right)} \cdot \frac{\hat{\chi}_{-i}-2}{\hat{\chi}_{-i}} . \\
& \frac{\left(\hat{\chi}_{i}+\frac{1 / \hat{\chi}_{-i}}{1-2 / \hat{\chi}_{-i}}\right)\left(\hat{\chi}_{i}-\left(2-1 / \hat{\chi}_{-i}\right)\right)\left(\hat{\chi}_{i}-1\right)}{\left(\hat{\chi}_{i}-1 / \hat{\chi}_{-i}\right)^{2}} .
\end{aligned}
$$

By Eqs. (22) and (28), we obtain

$$
\hat{\chi}_{i}\left(2-\hat{\chi}_{-i}\right)<1, \quad \forall i
$$

from which the interval of $\hat{\chi}_{i}$ is determined as

$$
\begin{cases}2-1 / \hat{\chi}_{-i}<\hat{\chi}_{i}<\max \left\{\hat{\chi}_{i}\right\} & \text { if } \hat{\chi}_{-i} \geq 2 \\ 2-1 / \hat{\chi}_{-i}<\hat{\chi}_{i}<\left(2-\hat{\chi}_{-i}\right)^{-1} & \text { if } 1<\hat{\chi}_{-i}<2 .\end{cases}
$$

where $\max \left\{\hat{\chi}_{i}\right\}=1+\left(\bar{\Pi} / \bar{\gamma}_{1}\right) \chi\left(\min \left\{\Lambda_{i}^{O F F}\right\}, \rho\right)$ and $\min \left\{\Lambda_{i}^{O F F}\right\}$ is the minimum possible $\Lambda_{i}^{O F F}$ available in the spectrum market.

By taking the first derivative of Eq. (29) and using Eq. (30), the maximal profit is determined as ${ }^{11}$

$$
\begin{aligned}
& \max F_{i}^{\left\{p_{i}=p_{-i}\right\}} \\
& \left\{\begin{array}{lll}
=0 & \text { at } \hat{\chi}_{i}=2-\frac{1}{\hat{\chi}_{-i}} \text { or }-\frac{1 / \hat{\chi}_{-i}}{1-2 / \hat{\chi}-i} & \text { if } \frac{3}{4}<\frac{1}{\hat{\chi}_{-i}}<1, \\
=0 & \text { at } \hat{\chi}_{i}=2-\frac{1}{\hat{\chi}_{-i}} & \text { if } \frac{1}{2}<\frac{1}{\hat{\chi}-i}<\frac{3}{4}, \\
>0 & \text { at } \hat{\chi}_{i}=\max \left\{\hat{\chi}_{i}\right\} & \text { if } X_{i}<\frac{1}{\hat{\chi}-i}<\frac{1}{2}
\end{array}\right.
\end{aligned}
$$

where $X_{i}:=\max \left\{0,1-\frac{\bar{\Pi}}{\bar{\gamma}_{1}} \chi\left(\min \left\{\Lambda_{i}^{O F F}\right\}, \rho\right)\right\}$. Therefore, to achieve positive profit, WSP $i$ operates its service only when

$$
X_{i}<\frac{1}{\hat{\chi}_{-i}^{*}}<\frac{1}{2} \text {, and } \hat{\chi}_{i}^{*}=1+\frac{\bar{\Pi}}{\bar{\gamma}_{1}} \chi\left(\min \left\{\Lambda_{i}^{O F F}\right\}, \rho\right) \text {. }
$$

Since Eq. (32) should hold for any $i$, we need to ensure that

$$
\begin{aligned}
& \frac{1}{\hat{\chi}_{i}}=\left\{1+\frac{\bar{\Pi}}{\bar{\gamma}_{1}} \chi\left(\min \left\{\Lambda_{i}^{O F F}\right\}, \rho\right)\right\}^{-1}<\frac{1}{2} \\
& \Leftrightarrow \quad \min \left\{\Lambda_{i}^{O F F}\right\}<\frac{\mu}{\beta}\left(1-\frac{\bar{\gamma}_{1}}{\bar{\Pi}} \cdot \frac{1}{\rho} \cdot \frac{\sum_{n=0}^{\alpha} \rho^{n} / n !}{\sum_{n=0}^{\alpha-1} \rho^{n} / n !}\right) .
\end{aligned}
$$

To make a fair and balanced profit, two WSPs would choose channels with the same quality such that

$$
\begin{aligned}
\Lambda^{O F F} & :=\Lambda_{1}^{O F F}=\Lambda_{2}^{O F F}<\frac{\mu}{\beta}\left(1-\frac{\bar{\gamma}_{1}}{\bar{\Pi}} \cdot \frac{1}{\rho} \cdot \frac{\sum_{n=0}^{\alpha} \frac{\rho^{n}}{n !}}{\sum_{n=0}^{\alpha-1} \frac{\rho^{n}}{n !}}\right), \\
\hat{\chi}^{*} & :=\hat{\chi}_{1}^{*}=\hat{\chi}_{2}^{*}=1+\frac{\bar{\Pi}}{\bar{\gamma}_{1}} \chi\left(\Lambda^{O F F}, \rho\right)
\end{aligned}
$$

from which the quality NE is determined as in Theorem 4.

Theorem 4. The NE of the quality competition exists only when $\hat{\chi}^{*}>2$, and is determined as $\left(u_{1}, u_{2}\right)=\left(u^{*}, u^{*}\right)$ where $u^{*}=2 /\left(\hat{\chi}^{*}+1\right)<2 / 3$.

Proof: The NE existence condition is directly given by Eq. (33), and the NE is derived by applying $\hat{\chi}^{*}=\hat{\chi}_{1}^{*}=\hat{\chi}_{2}^{*}$ to Eq. (27). The upperbound of $\Lambda^{O F F}$ in Eq. (34) leads to $\hat{\chi}^{*}>2$, and thus $u^{*}=2 /\left(\hat{\chi}^{*}+1\right)<2 / 3$.

\footnotetext{
${ }^{11}$ The derivation of Eq. (31), though omitted due to the space limit, is based on $\partial F_{i}^{\left\{p_{i}=p_{-i}\right\}} / \partial \hat{\chi}_{i}$ and the interval of $\hat{\chi}_{i}$ in Eq. (30).
}

TABLE I

THE NUMBER OF ALMOST IDENTICAL PAIRS OF CHANNELS.

\begin{tabular}{|c|c|c|c|c|c|c|c|}
\hline$N_{\text {ch }}$ & 20 & 30 & 40 & 50 & 60 & 70 & 80 \\
\hline$N_{\text {pairs }}$ & 0.71 & 1.6 & 2.9 & 4.6 & 6.6 & 9.2 & 11.9 \\
\hline
\end{tabular}

Corollary 3. At the NE of the quality competition, the $N E$ price becomes $p^{*}=p_{1}^{*}=p_{2}^{*}=\bar{\Pi}$.

Proof: The result directly comes from Theorem 2 and Corollary 1.

Corollary 4. At the NE of the quality competition, the profit of two WSPs becomes monotonically decreasing with $\Lambda^{O F F}$.

Proof: Applying $\Lambda_{1}^{O F F}=\Lambda_{2}^{O F F}=\Lambda^{O F F}$ and $\hat{\chi}_{1}=$ $\hat{\chi}_{2}=\hat{\chi}\left(\Lambda^{O F F}\right)$ to Eq. (29) yields

$$
F_{i}^{\left\{p_{i}=p_{-i}\right\}}\left(\Lambda^{O F F}\right)=\frac{1}{\bar{\gamma}_{1}} \cdot \frac{\chi\left(\Lambda^{O F F}, \rho\right)}{\chi\left(\Lambda^{O F F}, \rho / 2\right)} \cdot \frac{(\hat{\chi}-1)^{3}}{(\hat{\chi}+1)^{2}}
$$

where $\chi\left(\Lambda^{O F F}, \rho\right) / \chi\left(\Lambda^{O F F}, \rho / 2\right)$ is independent of $\Lambda^{O F F}$ (refer to Eq. (4)) and $\tilde{F}\left(\Lambda^{O F F}\right):=(\hat{\chi}-1)^{3} /(\hat{\chi}+1)^{2}$ is monotonically decreasing with $\Lambda^{O F F}$ since by combining Eq. (4), Eq. (28), and $\chi\left(\Lambda^{O F F}, \rho\right)>0$ we get

$$
\frac{\partial \tilde{F}\left(\Lambda^{O F F}\right)}{\partial \Lambda^{O F F}}=\frac{(\hat{\chi}-1)^{2}(\hat{\chi}+5)}{(\hat{\chi}+1)^{3}} \cdot \frac{\partial \hat{\chi}\left(\Lambda^{O F F}\right)}{\partial \Lambda^{O F F}}<0,
$$

which completes the proof.

\section{E. Achievability of Quality NE}

The difficulty of achieving the derived NE in Theorem 4 depends on the spectrum availability in a spectrum market. In case the market provides a plenty of channels to bid, it becomes more likely to find a pair of channels with the same $\left(u, \Lambda^{O F F}\right)$, i.e., identical channels. If there exist many such pairs, the two WSPs would try to bid a pair of identical channels with the minimum possible $\Lambda^{O F F}$ satisfying $u=2 /(\hat{\chi}+1)$ (which is the NE), due to Corollary 4 .

When the market availability is limited, it is difficult to find a pair of identical channels. From the practical point of view, however, $u$ and $\Lambda^{O F F}$ are estimated by PUs using the history of channel usage patterns. Since they are estimates, the market should allow some error margin on each feature. That is, even if two channels are not exactly identical, we should treat them the same as long as two $u$ 's (and two $\Lambda^{O F F}$ 's) are almost identical within an acceptable error margin. To investigate the achievability of (almost) identical channel pairs, a numerical analysis is performed while varying the number of channels $N_{c h}$ from 20 to 80 (in the step of 5) ) $^{12}$ and the number of almost identical channel pairs $N_{\text {pairs }}$ is counted with the error margin of $10 \%$ as shown in Table I. It is observed that $N_{\text {pairs }}$ increases exponentially as $N_{c h}$ grows, converging to $N_{\text {pairs }}=$ $e^{0.03 N_{c h}}$ for $N_{c h} \geq 40$. It is also measured that $N_{\text {pairs }} \geq 1.1$ (i.e., one or more pairs can be found) for $N_{c h} \geq 25$.

\footnotetext{
${ }^{12}$ Each channel is generated with exponential distribution with $\Lambda^{O F F}=$ $1 / 50$ and $\Lambda^{O N}=1 / 25$.
} 


\section{SERVice Differentiation Via Reimbursement}

In Sections $\mathrm{V}$ and $\mathrm{VI}$, we have considered price-driven service preference, i.e., customers choose a service based only on $p_{i}$, which is reasonable as long as WSPs set the same reimbursement rate $\beta$. On the contrary, if WSP $i$ offers differentiated service with its own reimbursement rate $\beta_{i}$ $\left(0<\beta_{i} \leq 1\right)$, the customers' service preference may be affected by both $p_{i}$ and $\beta_{i}$.

Suppose customers choose their service by the expected monetary cost per unit-time. Assuming that the amount of reimbursement is $\beta_{i}$ times the total service charge until eviction, the expected cost rate of a customer who has chosen WSP $i$, denoted by $\Omega_{i}$, is determined as

$$
\begin{aligned}
\Omega_{i}= & \int_{0}^{\infty}\left[\int_{y}^{\infty}\left(1-\beta_{i}\right) p_{i} f_{X}(x) d x+\right. \\
& \left.\int_{0}^{y} p_{i} f_{X}(x) d x\right] f_{Y}(y) d y=p_{i}\left(1-\frac{\beta_{i}}{1+\mu / \Lambda_{i}^{O F F}}\right),
\end{aligned}
$$

where $x$ denotes a customer's service time whose p.d.f. is given as $f_{X}(x)=\mu e^{-\mu x}$, and $y$ denotes the time remaining until the next OFF to ON transition from the customer's arrival whose p.d.f. is given as $f_{Y}(y)=\Lambda_{i}^{O F F} e^{-\Lambda_{i}^{O F F} y}$. Then, the customer chooses a service with smaller $\Omega_{i}$ that minimizes its cost rate, such as

$$
\Omega_{1} \underset{\text { WSP } 1}{\stackrel{\text { WSP }}{\gtrless}} \Omega_{2} \text {. }
$$

This suggests that WSP $i$ must announce not only its service price (i.e., $p_{i}$ ) but also its reimbursement rate (i.e., $\beta_{i}$ ) and the mean white space duration (i.e., $1 / \Lambda_{i}^{O F F}$ ), to help customers make the right choice.

As a special case, when $p_{1}=p_{2}$, Eq. (35) becomes

$$
\frac{\beta_{1}}{1+\mu / \Lambda_{1}^{O F F}} \underset{\text { WSP } 1}{\mathrm{WSP}} 2 \frac{\beta_{2}}{1+\mu / \Lambda_{2}^{O F F}},
$$

that is, customers should compare a normalized reimbursement rate $\beta_{i}\left(1+\mu / \Lambda_{1}^{O F F}\right)$ instead of the reimbursement rate $\beta_{i}$. Note that $\mu / \Lambda_{i}^{O F F}$ implies the average number of successive service sessions without eviction because

$$
\frac{\mu}{\Lambda_{i}^{O F F}}=\frac{1 / \Lambda_{i}^{O F F}}{1 / \mu}=\frac{\text { mean OFF period }}{\text { mean service time }}
$$

For example, even if $\beta_{1}>\beta_{2}$, customers may choose WSP 2 if $1+\mu / \Lambda_{2}^{O F F}$ is much smaller than $1+\mu / \Lambda_{1}^{O F F}$. As a result, customers face a dilemma: whether to choose WSP 1 with which service is not likely to be interrupted thanks to longer $\mu / \Lambda_{1}^{O F F}$, or to choose WSP 2 with which an eviction is more likely which is rewarded by reimbursement. This suggests a future research direction where customers are classified into two groups: one values service completion more than monetary reward, and vice versa.

As another special case, when $\Lambda^{O F F}=\Lambda_{1}^{O F F}=\Lambda_{2}^{O F F}$, Eq. (35) becomes

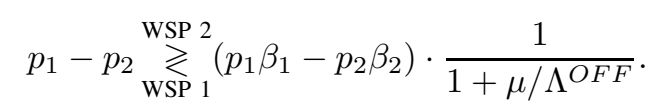

For example, assuming $p_{1} \geq p_{2}$, the following three cases are obtained:

$$
\begin{cases}\frac{p_{1}-p_{2}}{p_{1} \beta_{1}-p_{2} \beta_{2}} \underset{\text { WSP } 1}{\text { WSP } 2} \frac{1}{1+\mu / \Lambda^{\text {OFF }}} & \text { if } p_{1} \beta_{1}>p_{2} \beta_{2}, \\ \text { always WSP } 2 & \text { if } p_{1} \beta_{1} \leq p_{2} \beta_{2} \text { and } p_{1} \neq p_{2}, \\ \text { choose WSP } i \text { w.p. } 0.5 & \text { if } \beta_{1}=\beta_{2} \text { and } p_{1}=p_{2} .\end{cases}
$$

Deriving NE with heterogeneous reimbursement rates is possible by following the same steps in Sections V and VI.

\section{Evaluation of Super Wi-Fi MARKet Dynamics}

We now conduct extensive numerical analyses to provide insight into the dynamics of the Super Wi-Fi market. In the first test, we study under which condition the state decomposition approach provides a tolerable approximation error, by comparing the analytically derived profit with the observed profit from simulation. Next, we study the fundamental tradeoffs between the achieved profit at equilibrium, the customer arrival rate, and the channel leasing cost. Finally, we present the impact of the limited market availability on the two WSPs' achieved profit.

In all scenarios, common test parameters are set as follows: $C=5, B=1, \gamma_{2}=1, \beta=0.25$, and $\Lambda^{O N}=1 / 50$.

\section{A. Approximation Accuracy in State Decomposition}

We compare the profits given by the simulation and the analysis to derive the condition under which state decomposition performs reasonably well. In the simulation, we randomly generate 200 pairs of exponential ON and OFF periods and also emulate user arrivals and departures. A simulation is run by varying $\Lambda^{O F F}$ where the NE price and quality are applied, and each test condition is repeated 10 times to derive the average performance. Other simulation parameters are set as $\bar{\Pi}=2, \lambda=0.045, \mu=1 / 20$, and $\bar{\gamma}_{1}=0.1$.

From Fig. 6, one can see that the profit predicted by analysis gets fairly close to the actual achieved profit at a small $\Lambda^{O F F} / \mu$, and the gap between them gradually increases as $\Lambda^{O F F} / \mu$ grows. At $\Lambda^{O F F} / \mu=0.1$, the approximation error is found to be less than $9.5 \%$, which becomes around $15 \%$ at $\Lambda^{O F F} / \mu=0.15$. In case the tolerable error is less than $10 \%$, the state decomposition approach is effective for $\Lambda^{O F F} / \mu \leq 0.1$, implying that an OFF period, on average, can accommodate at least 10 consecutive user sessions. Note that this is a plausible scenario since DSA targets to reuse underutilized channels with relatively larger ON/OFF periods than customer arrivals/departures.

\section{B. Impact of Arrival Rate and Leasing Cost}

In Fig. 7(a), we plot the achieved profit of a WSP at its equilibrium while varying the arrival rate $\lambda$ (equivalently $\rho$ ). The leasing cost is also varied by testing three selected values of $\bar{\gamma}_{1}$. In this test, the simulation parameters are set as $\bar{\Pi}=1$, $\mu=1 / 5$, and $\Lambda^{O F F}=1 / 500$. It can be seen that as the arrival rate increases (i.e., $\rho \rightarrow 1$ ), the WSP achieves more profit due to the increased revenue. The profit also enhances as $\bar{\gamma}_{1}$ decreases, due to the less leasing cost by $L_{i}$. In Fig. 7(b), we also plot the quality equilibrium $u^{*}$ under the same test conditions. As the arrival rate increases, $u^{*}$ is monotonically 


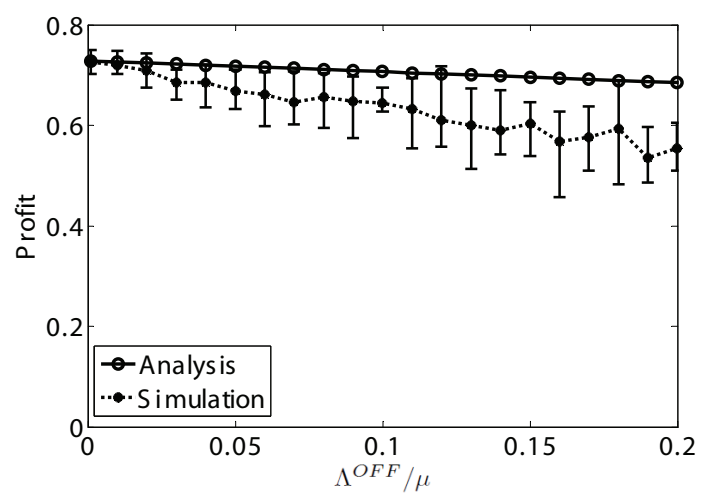

Fig. 6. Decomposition approximation accuracy

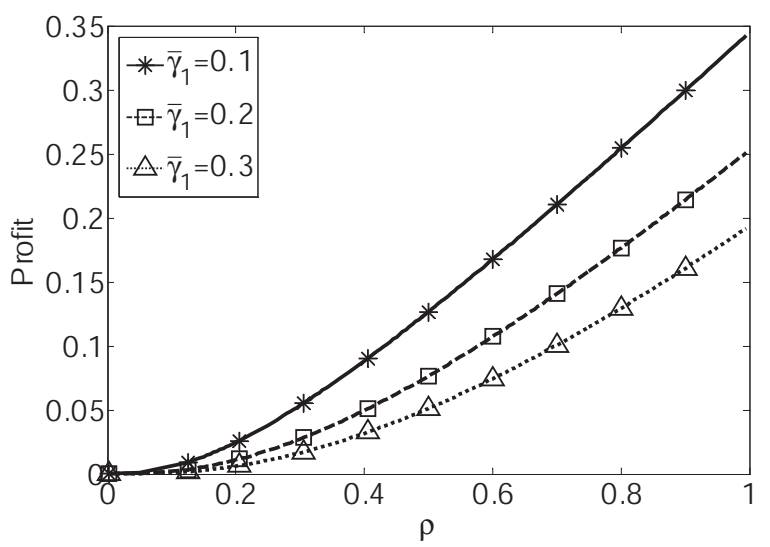

(a) Profit $F_{i}$ vs. arrival rate $\lambda$

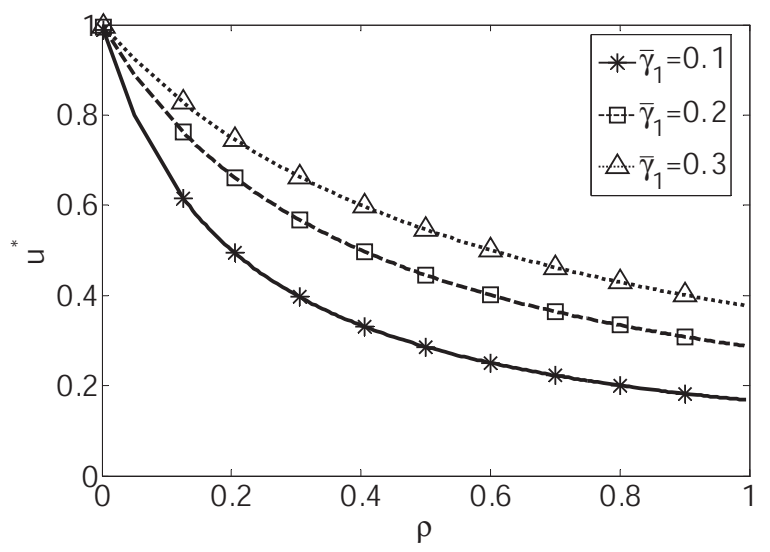

(b) Quality NE $u^{*}$ vs. arrival rate $\lambda$

Fig. 7. Fundamental tradeoffs in the duopoly market

decreasing because the WSP can overcome the leasing cost by accommodating more customers using a less busy channel (i.e., smaller $u^{*}$ ). Therefore, when $\rho \rightarrow 0$, the best strategy is to leave the market, i.e., $u^{*}=1$. At the same $\rho$, however, $u^{*}$ increases as $\bar{\gamma}_{1}$ increases, because it can compensate the increased leasing cost by leasing a less idle channel.

\section{CONCLUSION}

We modeled the competition between two WSPs in the duopoly Super Wi-Fi market as a hierarchical noncooperative game while considering time-varying spectrum availability. The model consists of a price sub-game for enticing customers to service and a quality full-game for leasing spectrum bands with heterogeneous quality. It has been shown that the price game has a unique NE provided that the marginal prices of the two WSPs are the same, and the quality game has a unique NE provided that two WSPs lease the same quality channels. Our extensive numerical analyses have demonstrated the fundamental tradeoffs amongst the customer arrival rate, channel dynamics, and eviction cost.

In future, we plan to investigate customer-centric Super WiFi service differentiation. For example, customers may favor a WSP with a smaller probability of eviction to ensure longer continuous transmissions. In addition, the reimbursement rate $\beta_{i}$ in Section VII may be reshaped as $\beta_{i k}$ where $k$ is the index of user classes (e.g., gold, silver, and bronze) such that users in a more expensive class may get more reimbursement.

\section{REFERENCES}

[1] H. Kim, J. Choi, and K. Shin, "Wi-Fi 2.0: Price and Quality Competitions of Duopoly Cognitive Radio Wireless Service Providers with Time-varying Spectrum Availability," in Proc. IEEE INFOCOM, April 2011.

[2] FCC, "Second Memorandum Opinion and Order," FCC 10-174, September 2010 .

[3] "IEEE 802.22 Working Group on Wireless Regional Area Networks." http://www.ieee802.org/22/.

[4] S. Deb, V. Srinivasan, and R. Maheshwari, "Dynamic Spectrum Access in DTV Whitespaces: Design Rules, Architecture and Algorithms," in Proc. ACM MobiCom, September 2009.

[5] P. Bahl, R. Chandra, T. Moscibroda, R. Murty, and M. Welsh, "White Space Networking with Wi-Fi like Connectivity," in Proc. ACM SIGCOMM, August 2009.

[6] A. Stirling, "White Spaces - the New Wi-Fi?," International Journal of Digital Television, vol. 1, no. 1, pp. 69-83, 2010.

[7] J. Chapin and W. Lehr, "The Path to Market Success for Dynamic Spectrum Access Technology," IEEE Commun. Mag., vol. 45, no. 5, pp. 96-103, 2007.

[8] H. Kim and K. Shin, "Admission and Eviction Control of Cognitive Radio Users at Wi-Fi 2.0 Hotspots," IEEE Trans. Mobile Comput., vol. 11, no. 11, pp. 1666-1677, 2012.

[9] H. Kim and K. Shin, "Efficient Discovery of Spectrum Opportunities with MAC-Layer Sensing in Cognitive Radio Networks," IEEE Trans. Mobile Comput., vol. 7, no. 5, pp. 533-545, 2008.

[10] S. Geirhofer, L. Tong, and B. Sadler, "Dynamic Spectrum Access in the Time Domain: Modeling and Exploiting White Space," IEEE Commun. Mag., vol. 45, no. 5, pp. 66-72, 2007.

[11] M. Buddhikot, "Understanding Dynamic Spectrum Access: Models, Taxonomy and Challenges," in Proc. IEEE DySPAN, April 2007.

[12] J. Jia and Q. Zhang, "Competitions and Dynamics of Duopoly Wireless Service Providers in Dynamic Spectrum Market," in Proc. ACM MobiHoc, 2008.

[13] L. Duan, J. Huang, and B. Shou, "Duopoly Competition in Dynamic Spectrum Leasing and Pricing," IEEE Trans. Mobile Comput., vol. 11, no. 11, pp. 1706-1719, 2012.

[14] K. Zhu, D. Niyato, P. Wang, and Z. Han, "Dynamic spectrum leasing and service selection in spectrum secondary market of cognitive radio networks," IEEE Trans. Wireless. Commun., vol. 11, no. 3, pp. 1136$1145,2012$.

[15] G. Kasbekar and S. Sarkar, "Spectrum Pricing Games with Bandwidth Uncertainty and Spatial Reuse in Cognitive Radio Networks," in Proc. ACM MobiHoc, September 2010.

[16] L. Duan, J. Huang, and B. Shou, "Investment and pricing with spectrum uncertainty: A cognitive operator's perspective," IEEE Trans. Mobile Comput., vol. 10, no. 11, pp. 1590-1604, 2011.

[17] D. Niyato and E. Hossain, "A Game-Theoretic Approach to Competitive Spectrum Sharing in Cognitive Radio Networks," in Proc. IEEE WCNC, 2007.

[18] G. S. Kasbekar, E. Altman, and S. Sarkar, "A Hierarchical Spatial Game over Licenced Resources," in Proc. GameNets, June 2009.

[19] Y. Wu, B. Wang, K. Liu, and T. Clancy, "A Multi-Winner Cognitive Spectrum Auction Framework with Collusion-Resistant Mechanisms," in Proc. IEEE DySPAN, October 2008. 
[20] S. Gandhi, C. Buragohain, L. Cao, H. Zheng, and S. Suri, "A General Framework for Wireless Spectrum Auctions," in Proc. IEEE DySPAN, 2007.

[21] C.-T. Chou and K. Shin, "Analysis of Adaptive Bandwidth Allocation in Wireless Networks with Multilevel Degradable Quality of Service," IEEE Trans. Mobile Comput., vol. 3, no. 1, pp. 5-17, 2004.

[22] Q.-A. Zeng and D. Agrawal, "Modeling and Efficient Handling of Handoffs in Integrated Wireless Mobile Networks," IEEE Trans. Veh. Technol., vol. 51, no. 6, pp. 1469-1478, 2002.

[23] M. Peha, "Mobility Patterns in Microcellular Wireless Networks," IEEE Trans. Mobile Comput., vol. 5, no. 1, pp. 52-63, 2006.

[24] F. Ashtiani, J. Salehi, and M. Aref, "Mobility Modeling and Analytical Solution for Spatial Traffic Distribution in Wireless Multimedia Networks," IEEE J. Sel. Areas Commun., vol. 21, no. 10, pp. 1699-1709, 2003.

[25] E. Zola and F. Barcelo-Arroyo, "Impact of Mobility Models on the Cell Residence Time in WLAN Networks," in Proc. IEEE Sarnoff Symposium, March 2009.

[26] S. Ghani and M. Schwartz, "A Decomposition Approximation for the Analysis of Voice/Data Integration," IEEE Trans. Commun., vol. 42, no. 7, pp. 2441-2452, 1994.

[27] R. Myerson, Game Theory: Analysis of Conflict. Cambridge, MA: Harvard University Press, 2002.

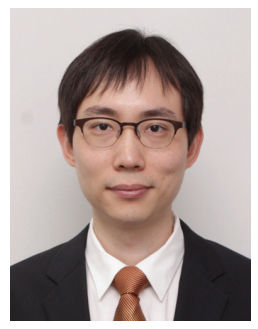

Hyoil Kim (M '10) is an assistant professor at the School of Electrical and Computer Engineering, the Ulsan National Institute of Science and Technology (UNIST), Ulsan, Korea, since August 2011. Before joining the UNIST, he was a postdoctoral researcher in the IBM T.J. Watson Research Center, Hawthorne, NY, USA in 2010-2011. He received his B.S. degree in Electrical Engineering from Seoul National University, Seoul, Korea in 1999, and M.S. and $\mathrm{Ph} . \mathrm{D}$. degrees in Electrical Engineering: Systems from the University of Michigan in 2005 and 2010, respectively. His research interests lie in wireless networking with an emphasis on cognitive radios (CRs), dynamic spectrum access (DSA), mobile cloud computing, and next-generation WLAN. He served as a TPC member of IEEE Globecom Wireless Networking Symposium (2011-2013), ICUFN (20122013), ACM WiNTECH 2013, and IEEE INFOCOM 2014, and also served as a publicity co-chair of ACM WiNTECH 2013.

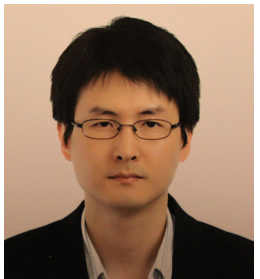

Jaehyuk Choi (M '07) received the $\mathrm{PhD}$ degree in Electrical Engineering and Computer Science from Seoul National University, Korea, in 2008. $\mathrm{He}$ is currently an assistant professor in the Department of Software Design \& Management at Gachon University, Seongnam, Korea. From 2009 to 2011, he was a postdoctoral research fellow in the Department of Electrical Engineering and Computer Science at the University of Michigan, Ann Arbor. He was a postdoctoral fellow in Brain Korea 21 at Seoul National University in 2008. His current research interests are in the areas of wireless/mobile networks with emphasis on wireless LAN/MAN/PAN, network management, next-generation mobile networks, cognitive radios, and wireless security. He is a member of the IEEE.

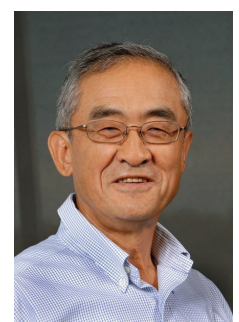

Kang G. Shin (F '92) is the Kevin \& Nancy O'Connor Professor of Computer Science in the Department of Electrical Engineering and Computer Science, The University of Michigan, Ann Arbor. His current research focuses on QoS-sensitive computing and networking as well as on embedded realtime and cyber-physical systems.

$\mathrm{He}$ has supervised the completion of $73 \mathrm{PhDs}$, and authored/coauthored more than 800 technical articles (more than 300 of these are in archival journals), a textbook and more than 20 patents or invention disclosures, and received numerous best paper awards, including the Best Paper Awards from the 2011 ACM International Conference on Mobile Computing and Networking (MobiCom'11), the 2011 IEEE International Conference on Autonomic Computing, the 2010 and 2000 USENIX Annual Technical Conferences, as well as the 2003 IEEE Communications Society William R. Bennett Prize Paper Award and the 1987 Outstanding IEEE Transactions of Automatic Control Paper Award. He has also received several institutional awards, including the Research Excellence Award in 1989, Outstanding Achievement Award in 1999, Distinguished Faculty Achievement Award in 2001, and Stephen Attwood Award in 2004 from The University of Michigan (the highest honor bestowed to Michigan Engineering faculty); a Distinguished Alumni Award of the College of Engineering, Seoul National University in 2002; 2003 IEEE RTC Technical Achievement Award; and 2006 Ho-Am Prize in Engineering (the highest honor bestowed to Korean-origin engineers). 